\title{
Hepatic Arterial Infusion Chemotherapy versus Sorafenib in Patients with Advanced Hepatocellular Carcinoma
}

\author{
Kazuomi Ueshima ${ }^{a}$ Sadahisa Ogasawara ${ }^{b, c} \quad$ Masafumi lkedad \\ Yutaka Yasui $^{\mathrm{C}}$ Takeshi Terashima ${ }^{\mathrm{f}, \mathrm{g}}$ Tatsuya Yamashita ${ }^{\mathrm{f}, \mathrm{g}}$ \\ Shuntaro Obi ${ }^{\text {h, }}$ Shinpei Sato ${ }^{i}$ Hiroshi Aikataj ${ }^{j}$ Takumi Ohmurak \\ Hidekatsu Kurodal Takamasa Ohkim Kengo Nagashiman ${ }^{\mathrm{m}}$ \\ Yoshihiko Ooka ${ }^{b}$ Masahiro Takita ${ }^{a}$ Masayuki Kurosaki ${ }^{\mathrm{e}}$ \\ Kazuaki Chayamaj Shuichi Kaneko ${ }^{f}$ Namiki Izumi $^{\mathrm{e}}$ Naoya Kato ${ }^{\text {b, c }}$ \\ Masatoshi Kudo ${ }^{a}$ Masao Omata ${ }^{\circ}$ p
}

a Department of Gastroenterology and Hepatology, Kindai University Faculty of Medicine, Osaka-Sayama, Japan; ${ }^{\mathrm{b}}$ Department of Gastroenterology, Graduate School of Medicine, Chiba University, Chiba, Japan; 'Translational Research and Development Center, Chiba University Hospital, Chiba, Japan; ${ }^{\mathrm{d}}$ Department of Hepatobiliary and Pancreatic Oncology, National Cancer Center Hospital East, Kashiwa, Japan; ${ }^{e}$ Department of Gastroenterology and Hepatology, Musashino Red Cross Hospital, Musashino, Japan; ${ }^{\mathrm{f} D e p a r t m e n t ~ o f ~}$ Gastroenterology, Kanazawa University Hospital, Kanazawa, Japan; ${ }^{9}$ Advanced Preventive Medical Sciences Research Center, Kanazawa University, Kanazawa, Japan; ${ }^{\text {h}}$ Third Department of Internal Medicine, Teikyo University School of Medicine, Ichihara, Japan; iDepartment of Gastroenterology and Hepatology, Kyoundo Hospital of the Sasaki Institute, Tokyo, Japan; ${ }^{j}$ Department of Gastroenterology and Metabolism, Hiroshima University Hospital, Hiroshima, Japan; ' Department of Gastroenterology, Sapporo Kosei General Hospital, Sapporo, Japan; 'Division of Hepatology, Department of Internal Medicine, Iwate Medical University, Morioka, Japan; ${ }^{\mathrm{m}}$ Department of Gastroenterology, Mitsui Memorial Hospital, Tokyo, Japan; ${ }^{n}$ Research Center for Medical and Health Data Science, The Institute of Statistical Mathematics, Tokyo, Japan; ' Yamanashi Prefectural Central Hospital, Kofu,

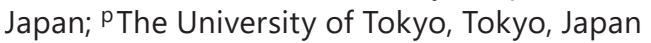

\section{Keywords}

Hepatic arterial infusion chemotherapy - Sorafenib - Advanced hepatocellular carcinoma .

Macrovascular invasion

\section{Abstract}

Background: Prior to the approval of sorafenib, hepatic arterial infusion chemotherapy (HAIC) was offered to patients with advanced hepatocellular carcinoma (HCC) in East Asia, particularly Japan. According to the Japanese guidelines, HAIC is recommended as one of the treat-

K. Ueshima, S. Ogasawara, and M. Ikeda contributed equally to this work. 
ment options in patients without extrahepatic metastasis (EHM). Methods: The present cohort study compared the use of HAIC and sorafenib on outcomes of patients with advanced HCC. Consecutive patients with advanced HCC who received HAIC or sorafenib as a first-line systemic therapy were enrolled from 10 Japanese institutions. The primary outcomes were overall survival (OS) in patients with macrovascular invasion (MVI), but without EHM, and OS in patients without both MVI and EHM. Results: Between 2009 and 2016, 2,006 patients were enrolled (541 HAIC patients, 1,465 sorafenib patients). After propensity score matching, the OS of patients with MVI but without EHM was significantly longer in the HAIC group compared with the sorafenib group (10.1 vs. 9.1 months for the HAIC and sorafenib groups, respectively; $n=170$ for each group; hazard ratio [HR] 0.668; 95\% confidence interval [95\% Cl] 0.475-0.935; $p=0.018)$. There was no significant difference in OS between patients without both $\mathrm{MVI}$ and EHM (12.2 vs. 15.4 months for the HAIC and sorafenib groups, respectively; $n=76$ in each cohort after propensity score matching; HR $1.227 ; 95 \% \mathrm{Cl} 0.699-2.155 ; p=0.475)$. Conclusion: HAIC is a potential front-line treatment choice in a subpopulation of patients with advanced HCC with MVI but without EHM.

(c) 2020 The Author(s)

Published by S. Karger AG, Basel

\section{Introduction}

Hepatocellular carcinoma (HCC) is a leading cause of cancer-related death, with 850,000 new cases reported per year worldwide $[1,2]$. Two randomized placebo-controlled phase III studies reported sorafenib as the first systemic agent approved for use in patients with advanced HCC, and was the only drug used for nearly a decade [3, 4]. Recently, lenvatinib has shown clinical benefits and been approved by the US Food and Drug Administration for use as a front-line medication [5]. Sorafenib remains a first-line drug, and shows an improvement in median overall survival (OS) of 2.8 months compared with placebo (10.7 vs 7.9 months; hazard ratio [HR] $0.69 ; p<0.001$ ), despite a low response rate of $2 \%$ and limitations such as modest survival advantages and low response rates [3, 4].

Prior to the approval of sorafenib, hepatic arterial infusion chemotherapy (HAIC) was a common suboptimal therapy for advanced HCC in East Asia, particularly in Japan [6, 7]. Current Japanese guidelines recommend HAIC as a treatment option in patients with advanced HCC without extrahepatic metastasis (EHM) [8]. This treatment allows direct delivery of chemotherapeutic agents into tumors feeding hepatic arteries at increased local concentration, and provides strong antitumor effects with lower systemic toxicity via a first-pass effect in the liver [9]. In their study, Nouso et al. [10] reported the efficacy of HAIC of 5-fluorouracil (5FU) and cisplatin (CDDP) for advanced HCC with or without macrovascular invasion (MVI) using the Nationwide Survey of Primary Liver Cancer in Japan, which collected initial treatment data from each patient. The median OS was longer in patients who underwent HAIC compared with those who did not receive active treatment. Several previous retrospective studies using small sample sizes showed high response rates, with long OS and tolerable side effects, particularly in patients with advanced HCC, including those with MVI [11-14]. The results of the SILIUS study, which compared sorafenib plus HAIC of 5FU + CDDP versus sorafenib in a randomized, open label, phase III study, showed no significant difference in OS between the two groups. However, subanalyses of the SILIUS study indicated that sorafenib plus HAIC showed survival benefits in patients with advanced HCC with main portal vein invasion [15]. A recent phase III, randomized, open label trial in China indicated that sorafenib plus HAIC of oxaliplatin, 5FU, and leucovorin (FOLFOX) improved OS compared with sorafenib in patients with advanced HCC with portal vein invasion [16]. On the other hand, few studies have directly compared sorafenib and HAIC in well-designed clinical trials using large 
numbers of advanced HCC patients with MVI. Although sorafenib plus HAIC treatment was shown to prolong OS compared with sorafenib alone in a limited population $[15,16]$, the survival benefits of HAIC directly compared with those of sorafenib have not yet been fully clarified in patients with advanced HCC. Therefore, the present study compared the survival outcome of HAIC and sorafenib in a real-world setting using a large retrospective cohort treated with HAIC and sorafenib during the same period. The main objective of the present study was to clarify the survival outcome of HAIC compared with sorafenib in patients with advanced HCC.

\section{Patients and Methods}

We set up an organizing committee to plan this retrospective study. This committee created the study protocol, which included clinical parameters and statistical analyses, such as predefined propensity scorematching methods and risk factors. Ethical approval was obtained from the Research Ethics Committee or an equivalent committee at all institutions. After approval of the protocol, predefined clinical parameters were corrected for the electronic data capture system. The study data were locked on December 10, 2018 and analyzed by an independent biostatistician (K.N.).

\section{Study Design, Inclusion/Exclusion Criteria, and Endpoints}

This retrospective cohort study was performed across 10 Japanese institutions (the Kindai University Faculty of Medicine, the Chiba University, the National Cancer Center Hospital East, the Musashino Red Cross Hospital, the Kanazawa University, the Kyoundo Hospital of the Sasaki Institute, the Hiroshima University Hospital, the Sapporo Kosei General Hospital, the Iwate Medical University, and the Mitsui Memorial Hospital). Eligible patients were those aged 20 years or older with advanced HCC who received HAIC or sorafenib treatment. We excluded patients who received combination therapies with HAIC or sorafenib (e.g., sorafenib combined with TACE, HAIC combined with sorafenib, sequential therapy of HAC and sorafenib, or HAIC combined with radiation therapy). Patients who satisfied the inclusion and exclusion criteria were enrolled consecutively and their data were entered into a dedicated database.

The predefined clinical parameters included: baseline demographic data (gender, age, etiology, Child-Pugh class, radiological assessment, alpha-fetoprotein [AFP] levels, and treatment prior to initiation of HAIC or sorafenib), HAIC regimen, initial dose of sorafenib, date of HAIC or sorafenib discontinuation, reasons for discontinuation of HAIC or sorafenib, and date of death or last follow-up. In the present study, we classified advanced HCC patients with MVI into two categories. The first category, major MVI, was defined as any of the following findings at the time of baseline radiological assessments; Vp 3 (primary branch portal vein invasion); Vp4 (main portal vein invasion); Vv 3 (inferior vena cava invasion); B3 (primary branch bile duct invasion); or B4 (main bile duct invasion). Minor MVI was the category of other cases of MVI.

We subdivided the enrolled patients into four groups according to the presence or absence of MVI and EHM: cohort 1, MVI+ and EHM-; cohort 2, MVI- and EHM-; cohort 3, MVI+ and EHM+; and cohort 4, MVI- and EHM+. The primary endpoint of the study was comparison of OS following HAIC and sorafenib treatment in patients with advanced HCC without EHM (cohorts 1 and 2). The preplanned secondary endpoints included comparison of OS following HAIC and sorafenib treatment in patients with advanced HCC with EHM (cohorts 3 and 4), evaluating outcomes of HAIC between different treatment regimens, reasons for discontinuation of both HAIC and sorafenib, and conversion rates of post-therapies to either HAIC or sorafenib.

\section{Statistical Analysis}

After data locking, we excluded patients with a Child-Pugh score $>7$ and started sorafenib $<400 \mathrm{mg}$, and fixed the data set of analyses. OS was calculated from the date of starting treatment to death from any cause. The censoring date was defined as the date of the last follow-up. Time to treatment failure (TTF) was defined as the date of starting treatment to discontinuing the treatment due to any cause. The censoring date was defined as the date of the last follow-up.

For the primary analyses, the OS of patients in the HAIC and sorafenib groups in cohorts 1 and 2 were compared using stratified log-rank tests in propensity score-matched samples. Propensity scores were estimated using logistic regression models with the following preplanned covariates: cohort 1, MVI (major vs. 


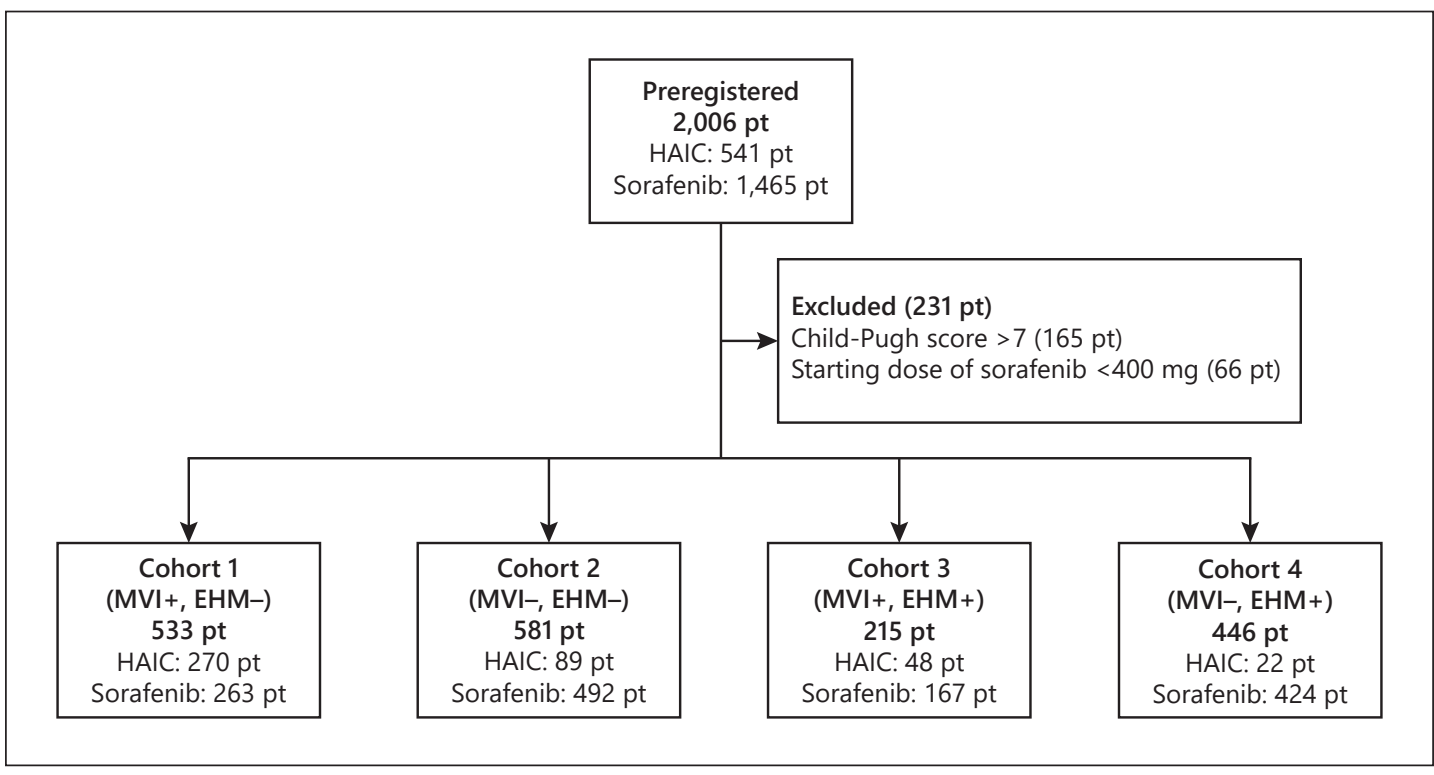

Fig. 1. Patient flow of the study. pt, patients.

minor), size of intrahepatic lesion ( $\leq 50$ vs. $>50 \mathrm{~mm}$ ), number of intrahepatic lesions ( $\leq 3$ vs. $>3$ ), Child-Pugh class (A vs. B), and AFP ( $\leq 400$ vs. $>400 \mathrm{ng} / \mathrm{mL}$ ); and cohort 2 , size of intrahepatic lesion ( $\leq 50 \mathrm{vs.}>50 \mathrm{~mm}$ ), number of intrahepatic lesions ( $\leq 7$ vs. $>7$ ), Child-Pugh class (A vs. B), AFP ( $\leq 400 \mathrm{vs.}>400 \mathrm{ng} / \mathrm{mL}$ ), and number of TACEs $(\leq 2$ or $>2$ ). Propensity score matching was performed using nearest-neighbor matching and 1:1 matching without replacement using caliper widths of 0.05 . Covariate balance was assessed using standardized mean differences for the propensity-matched cohorts. Kaplan-Meier plots of medians (with 95\% confidence interval; 95\% CI) were used to estimate OS, and HRs were estimated using Cox regression models after propensity score matching.

For secondary analyses, the OS of patients in the HAIC and sorafenib groups in cohorts 3 and 4 were compared using stratified log-rank tests in propensity score-matched samples. The preplanned covariates for cohorts 3 and 4 were as follows: cohort 3, MVI (major vs. minor), size of intrahepatic lesion ( $\leq 50$ vs. $>50$ $\mathrm{mm}$ ), number of intrahepatic lesions ( $\leq 3 \mathrm{vs.}>3$ ), Child-Pugh class (A vs. B), AFP ( $\leq 400 \mathrm{vs.}>400 \mathrm{ng} / \mathrm{mL}$ ), and number of EHM ( 1 vs. $>2$ ); and cohort 4 , size of intrahepatic lesion ( $\leq 50 \mathrm{vs}$. $>50 \mathrm{~mm}$ ), number of intrahepatic lesions ( $\leq 7$ vs. $>7$ ), Child-Pugh class (A vs. B), AFP ( $\leq 400$ vs. $>400 \mathrm{ng} / \mathrm{mL}$ ), and number of EHM ( 1 vs. $>2$ ). The OS of patients treated using HAIC was compared between each regimen using Cox regression models. The reasons for discontinuation of treatments and post-therapies were summarized as numbers and frequencies.

The significance level for the statistical analyses was set at $5 \%$ for two-sided tests. All statistical analyses were described in the statistical analysis plan that was finalized prior to locking the database, and were performed using SAS version 9.4 (SAS Institute, Cary, NC, USA) and Stata version 15 (StataCorp LLC, College Station, TX, USA).

\section{Results}

\section{Patient Flow}

A total of 2,006 patients (1,465 sorafenib patients, 541 HAIC patients) with advanced HCC were enrolled from 10 Japanese institutions. Figure 1 shows the patient flow of the study. We excluded patients with a Child-Pugh score $>7$ at baseline $(n=165)$ and a starting dose of $<400 \mathrm{mg}$ of sorafenib $(n=66)$. Of the 1,775 eligible patients, $533(30.0 \%), 581(32.7 \%), 215$ $(12.1 \%)$, and $446(26.3 \%)$ were enrolled in cohorts $1,2,3$, and 4 , respectively. 


\begin{tabular}{l|l}
\hline Liver Cancer 2020;9:583-595 \\
\hline DOI: 10.1159/000508724 & $\begin{array}{l}\text { @ 2020 The Author(s). Published by S. Karger AG, Basel } \\
\text { www.karger.com/lic }\end{array}$ \\
\hline
\end{tabular}

Table 1. Baseline characteristics of patients with advanced HCC in cohort 1 (patients with MVI and without EHM)

\begin{tabular}{|c|c|c|c|c|c|c|}
\hline & \multicolumn{3}{|l|}{ Unmatched } & \multicolumn{3}{|l|}{ Matched } \\
\hline & $\begin{array}{l}\text { HAIC } \\
(n=270)\end{array}$ & $\begin{array}{l}\text { sorafenib } \\
(n=263)\end{array}$ & SMD & $\begin{array}{l}\text { HAIC } \\
(n=170)\end{array}$ & $\begin{array}{l}\text { sorafenib } \\
(n=170)\end{array}$ & SMD \\
\hline Mean age, years & 67.1 & 69.0 & 0.210 & 67.3 & 68.9 & 0.175 \\
\hline Male gender & $214(79.3)$ & 207 (78.7) & -0.0014 & $141(82.0)$ & $130(75.6)$ & -0.157 \\
\hline HCV positive & $130(48.1)$ & $122(46.4)$ & 0.035 & $90(52.3)$ & $75(43.6)$ & 0.175 \\
\hline HBV positive & $62(23.0)$ & $56(21.3)$ & 0.040 & $35(20.3)$ & $40(23.3)$ & -0.070 \\
\hline Alcohol abuse & $40(14.9)$ & $32(12.2)$ & 0.079 & $29(17.0)$ & $20(11.6)$ & 0.153 \\
\hline Major MVI ${ }^{1}$ & $184(68.1)$ & $145(55.1)$ & 0.270 & $106(61.6)$ & $106(61.6)$ & 0.000 \\
\hline Size of intrahepatic lesion $>50 \mathrm{~mm}^{1}$ & $62(23.2)$ & $87(33.1)$ & -0.221 & $47(27.3)$ & $47(27.3)$ & 0.000 \\
\hline Number of intrahepatic lesions $>3^{1}$ & $165(62.0)$ & $171(65.0)$ & -0.0062 & $114(66.3)$ & $114(66.3)$ & 0.000 \\
\hline Child-Pugh class $\mathrm{A}^{1}$ & $157(63.1)$ & $221(84.0)$ & 0.490 & $133(77.3)$ & $133(77.3)$ & 0.000 \\
\hline $\mathrm{AFP}>400 \mathrm{ng} / \mathrm{mL}^{1}$ & $142(52.6)$ & $134(51.0)$ & 0.033 & $98(57.0)$ & $98(57.0)$ & 0.000 \\
\hline Number of TACEs $>0$ & $116(44.1)$ & $130(49.8)$ & -0.114 & $77(45.3)$ & $88(51.5)$ & -0.124 \\
\hline
\end{tabular}

Data are presented as the mean or $n(\%)$. AFP, alfa-fetoprotein; HAIC, hepatic arterial infusion chemotherapy; HBV, hepatitis B virus; HCV, hepatitis C virus; MVI, macrovascular invasion; SMD, standardized mean difference; TACE, transarterial chemoembolization.

${ }^{1}$ Major MVI, size of intrahepatic lesion, number of intrahepatic lesions, Child-Pugh class, and AFP were matched in cohort 1.

Table 2. Baseline characteristics of advanced HCC patients with cohort 2 (patients without both MVI and EHM)

\begin{tabular}{|c|c|c|c|c|c|c|}
\hline & \multicolumn{3}{|l|}{ Unmatched } & \multicolumn{3}{|l|}{ Matched } \\
\hline & $\begin{array}{l}\text { HAIC } \\
(n=89)\end{array}$ & $\begin{array}{l}\text { sorafenib } \\
(n=492)\end{array}$ & SMD & $\begin{array}{l}\text { HAIC } \\
(n=76)\end{array}$ & $\begin{array}{l}\text { sorafenib } \\
(n=76)\end{array}$ & SMD \\
\hline Mean age, years & 70.0 & 71.3 & 0.160 & 70.3 & 71.6 & 0.160 \\
\hline Male gender & $69(77.5)$ & $384(78.0)$ & 0.013 & $60(78.9)$ & $57(75.0)$ & -0.094 \\
\hline HCV positive & $51(57.3)$ & $303(61.6)$ & -0.087 & $42(55.3)$ & $45(59.2)$ & -0.080 \\
\hline HBV positive & $14(15.7)$ & $60(12.2)$ & 0.102 & $12(15.8)$ & $13(17.1)$ & -0.035 \\
\hline Alcohol abuse & $6(6.7)$ & $32(6.5)$ & 0.010 & $6(7.9)$ & $4(5.3)$ & 0.106 \\
\hline Size of intrahepatic lesion $>50 \mathrm{~mm}^{1}$ & $11(12.8)$ & $66(13.5)$ & -0.020 & $11(14.5)$ & $16(21.1)$ & -0.173 \\
\hline Number of intrahepatic lesions $>7^{1}$ & $51(59.3)$ & $219(44.6)$ & 0.297 & $46(60.5)$ & $44(57.9)$ & 0.054 \\
\hline Child-Pugh class $A^{1}$ & $53(63.9)$ & $428(87.5)$ & 0.574 & $51(67.1)$ & $51(67.1)$ & 0.000 \\
\hline $\mathrm{AFP}>400 \mathrm{ng} / \mathrm{mL}^{1}$ & $34(38.2)$ & $167(33.9)$ & 0.089 & $32(42.1)$ & $32(42.1)$ & 0.000 \\
\hline Number of TACEs $>2^{1}$ & $42(51.9)$ & $185(38.5)$ & 0.272 & $39(51.3)$ & $41(53.9)$ & -0.053 \\
\hline
\end{tabular}

Data are presented as the mean or $n(\%)$. AFP, alfa-fetoprotein; EHM, extrahepatic metastasis; HAIC, hepatic arterial infusion chemotherapy; HBV, hepatitis B virus; HCV, hepatitis C virus; MVI, macrovascular invasion; SMD, standardized mean difference; TACE, transarterial chemoembolization.

${ }^{1}$ Size of intrahepatic lesion, number of intrahepatic lesions, Child-Pugh class, AFP, and number of TACEs were matched in cohort 2 .

\section{Outcomes of HAIC and Sorafenib Treatment in Advanced HCC Patients without EHM}

Tables 1 and 2 show the unmatched and matched baseline characteristics of cohorts 1 and 2, respectively. The most frequent underlying etiology of HCC was hepatitis C virus, followed by hepatitis B virus and alcohol abuse. Rates of Child-Pugh A were higher in the sorafenib group compared with the HAIC group in both cohorts. After propensity score matching, absolute values of standardized mean differences of the baseline characteristics were smaller than 0.25 , and the baseline characteristics were balanced.

Kaplan-Meier survival curves of OS in cohorts 1 and 2 (after propensity score matching) are shown in Figure 2. Among the 340 advanced HCC patients in cohort 1, the OS in the HAIC 


\begin{tabular}{ll|l}
\cline { 2 - 2 } Liver Cancer & \multicolumn{2}{l}{ Liver Cancer 2020;9:583-595 } \\
\cline { 2 - 2 } & $\begin{array}{l}\text { DOI: 10.1159/000508724 } 2020 \text { The Author(s). Published by S. Karger AG, Basel } \\
\text { www.karger.com/lic }\end{array}$ \\
\cline { 2 - 2 } & Ueshima et al.: HAIC versus Sorafenib in Patients with Advanced HCC
\end{tabular}

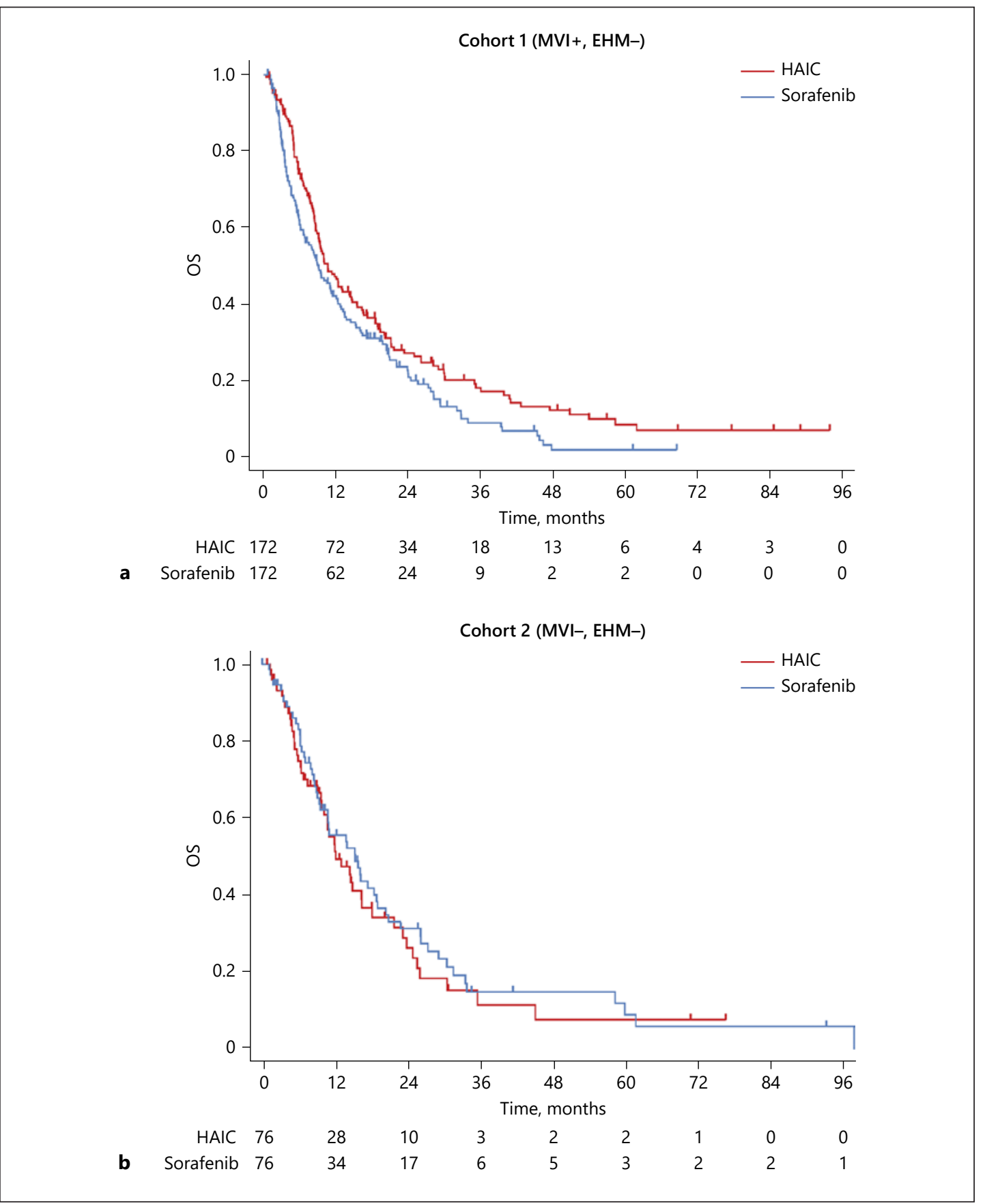

Fig. 2. OS of patients in cohort 1 (a) and cohort 2 (b) with advanced HCC who received HAIC and sorafenib.

group was significantly longer than that of the sorafenib group (Fig. 2a; HAIC, 10.6 months [95\% CI 9.1-14.3] vs. sorafenib, 9.1 months [95\% CI 6.8-12.0]; HR 0.667 [95\% CI 0.4750.935]; $p=0.018$ ). The median TTF for HAIC and sorafenib in cohort 1 was 2.8 months $(95 \%$ CI 2.4-3.0) and 2.5 months (95\% CI 1.8-2.9), respectively (HR 0.957 [95\% CI 0.772-1.185]; $p=0.683$ ). In contrast, there was no significant difference between HAIC and sorafenib treatment in cohort 2 (Fig. 2b; HAIC, 12.2 months [95\% CI 9.9-16.5] vs. sorafenib, 15.4 months [95\% CI 9.7-19.1]; HR 1.227 [95\% CI 0.699-2.155]; $p=0.475$ ). The median TTF for sorafenib 


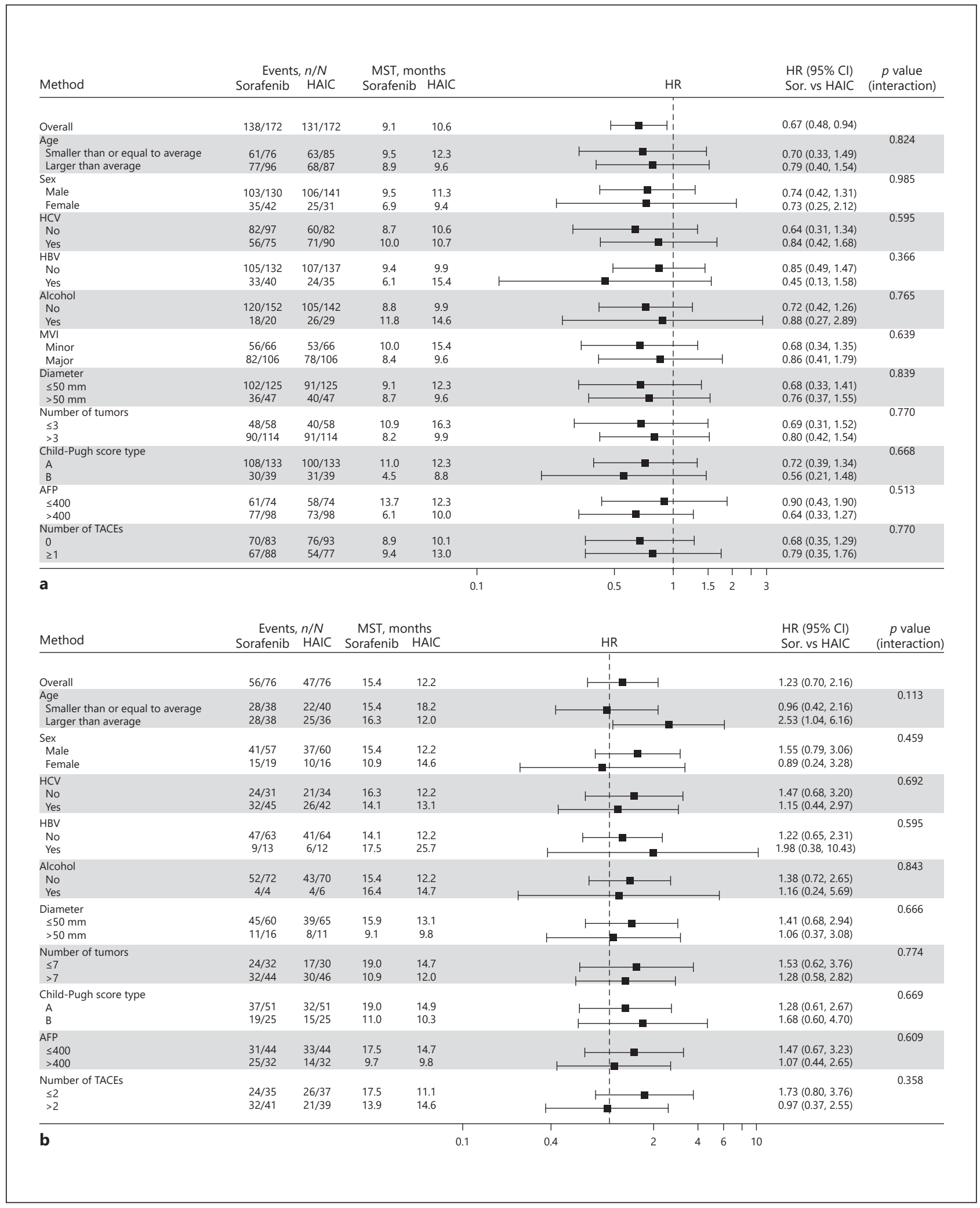

Fig. 3. Subgroup analyses of patients in cohort 1 (a) and cohort 2 (b) with advanced HCC who received HAIC and sorafenib. 
was significantly longer than that of HAIC in cohort 2 (HAIC: 2.7 months [95\% CI 2.2-3.6]; sorafenib: 4.9 months [95\% CI 3.0-6.8]; HR 1.563 [95\% CI 1.126-2.165]; $p=0.007$ ). Figure 3 shows subgroup analyses of cohorts 1 and 2 . The improvement in OS with HAIC was maintained in all subgroup analyses in patients with advanced HCC with MVI (cohort 1, Fig. 3a).

\section{HAIC and Sorafenib Outcomes in Patients with Advanced HCC with EHM}

The baseline characteristics of cohorts 3 and 4 are shown in online supplementary Tables 1 and 2 (for all online suppl. material, see www.karger,com/doi/10.1159/000508724), respectively. Among 215 patients with advanced HCC in cohort 3, 167 (77.7\%) received sorafenib. In cohort 4, the majority of patients (424 of 446, 95.1\%) also received sorafenib. While obvious imbalances were observed between the two groups in both cohorts, we compared the OS between HAIC and sorafenib treatment after propensity score matching (online suppl. Fig. 1). OS following HAIC treatment was significantly longer than that following sorafenib treatment in cohort 3 (online suppl. Fig. 1A; HAIC, 6.5 months [95\% CI 4.7-9.5] vs. sorafenib, 5.3 months [95\% CI 3.7-5.8]; HR 0.500 [95\% CI 0.250-1.000]; $p=0.046$ ). The median TTF for HAIC and sorafenib was 2.6 months (95\% CI 1.3-3.2) and 2.0 months (95\% CI 1.33.5), respectively (HR 0.820 [95\% CI 0.527-1.275]; $p=0.374$ ). On the other hand, there was no significant difference between HAIC and sorafenib treatment in cohort 4 (online suppl. Fig. 1B; HAIC, 6.3 months [95\% CI 1.7-10.9] vs. sorafenib, 9.0 months [95\% CI 4.2-11.8]; HR 1.125 [95\% CI 0.434-2.915]; $p=0.808$ ). The median TTF was also similar for HAIC and sorafenib (HAIC: 2.3 months [95\% CI 0.9-2.7]; sorafenib: 2.3 months [95\% CI 1.1-3.9]; HR 1.094 [95\% CI 0.566-2.114]; $p=0.789$ ).

\section{Subgroup Analyses of Patients with Advanced HCC According to MVI}

Online supplementary Table 3 indicates subgroup analyses of advanced HCC patients with MVI and the absence or presence of EHM (cohort 1 and 3). The median OS for HAIC was significantly longer in those with minor MVI and VP $<3$ in cohort 1 compared with sorafenib. On the other hand, the median OS for HAIC was significantly longer in those with major MVI and in the VP $\geq 3$ population in cohort 3 compared with sorafenib.

\section{Comparison of Outcomes of HAIC between Each Regimen}

We compared the outcomes of the HAIC regimens between 5FU + CDDP, 5FU + interferon (IFN), and CDDP alone. Among the 429 patients who received HAIC in the present study, $64.3 \%$ (276 patients), 18.6\% (80 patients), and 15.9\% (68 patients) received 5FU + CDDP, $5 F U+$ IFN, and CDDP alone, respectively. Online supplementary Figure 2 shows Kaplan-Meier curves of OS in patients with advanced HCC who received HAIC in the present study. The median OS values for patients receiving 5FU + CDDP, 5FU + IFN, and CDDP alone were 10.5 (95\% CI 9.2-12.3), 12.0 (95\% CI 9.0-14.6), and 7.8 (95\% CI 6.3-12.1) months, respectively ( $p=0.759$; online suppl. Fig. 2A). Analysis of the subpopulation of patients with advanced HCC with MVI and without EHM showed median OS values for patients receiving $5 \mathrm{FU}+\mathrm{CDDP}, 5 \mathrm{FU}$ + IFN, and CDDP alone of 10.5 (95\% CI 8.9-12.9), 11.2 (95\% CI 8.5-16.5), and 7.8 (95\% CI 6.3-14.0) months, respectively ( $p=0.948$; online suppl. Fig. 2B). Multivariate analyses confirmed that each HAIC regimen had no impact on survival in the present cohort (online suppl. Tables 4 and 5).

\section{Reasons for Discontinuation of Treatments and Post-Therapies}

Among 429 patients who received HAIC treatment, 256 (50.7\%) and 111 (25.9\%) patients discontinued treatment due to disease progression and adverse events, respectively. Likewise, among 1,346 patients who received sorafenib and satisfied the eligibility criteria, 822 (61.1\%) and $399(29.6 \%)$ patients discontinued treatment due to disease progression and adverse 
Table 3. Cause of treatment discontinuation (incidence rate $>2 \%$ )
Ueshima et al.: HAIC versus Sorafenib in Patients with Advanced HCC

\begin{tabular}{lcc}
\hline & \multicolumn{2}{l}{ Eligible patients $(n=1,775), n(\%)$} \\
\cline { 2 - 3 } & HAIC & $\begin{array}{l}\text { sorafenib } \\
(n=429)\end{array}$ \\
& $111(25.9)$ & $399(29.6)$ \\
\hline Any adverse event & $27(6.7)$ & 0 \\
Catheter-related adverse events & $20(4.7)$ & $77(5.7)$ \\
Liver failure & $3(0.7)$ & $49(3.6)$ \\
General disorders & 0 & $30(2.2)$ \\
Rash & $10(2.3)$ & 0 \\
Catheter occlusion & 0 & $30(2.2)$ \\
Hand-foot-skin syndrome & $1(0.2)$ & $28(2.1)$ \\
Anorexia & $9(2.1)$ & $9(0.7)$ \\
Renal failure & $9(2.1)$ & $2(0.1)$ \\
Abdominal pain & & \\
\hline
\end{tabular}

events, respectively. Table 3 shows the reasons for the discontinuation of treatments due to adverse events in both the HAIC and sorafenib groups. The most common reasons for discontinuing HAIC treatment were liver failure (4.7\%), catheter occlusion $(2.3 \%)$, renal failure (2.1\%), and abdominal pain (2.1\%). A total of 27 patients (6.7\%) discontinued HAIC treatment due to catheter-related adverse events. On the other hand, the most common reasons for discontinuation of sorafenib treatment were liver failure (5.7\%), general disorders $(3.6 \%)$, rash (2.2\%), hand-foot-skin reaction (2.2\%), and anorexia. Online supplementary Tables 6 , 7,8 , and 9 show the post-treatments after HAIC or sorafenib in cohorts $1,2,3$, and 4, respectively (both unmatched and matched cohorts). In cohort 1, the conversion rates from HAIC to sorafenib and sorafenib to HAIC were 23.5 and $24.2 \%$, respectively (matched cohort). The median post-treatment survival of HAIC to sorafenib and sorafenib to HAIC were 12.0 months (95\% CI 6.0-18.0) and 10.2 months (95\% CI 8.4-14.2), respectively. Similarly, 19.7 and 18.3\% of cohort 2 patients were converted from HAIC to sorafenib, and sorafenib to HAIC, respectively. The median post-treatment survival times of HAIC to sorafenib and sorafenib to HAIC were 18.9 months (95\% CI 4.4-40.5) and 10.6 months (4.0-19.8), respectively.

\section{Discussion}

The primary outcome of the present study showed that HAIC treatment had greater survival benefits compared with sorafenib in patients with advanced HCC with MVI (without EHM; cohort 1). On the other hand, there was no significant difference in OS between HAIC and sorafenib treatment in patients without MVI and EHM (cohort 2). To the best of our knowledge, this is the first study to demonstrate that HAIC treatment significantly prolonged OS compared with sorafenib treatment in a subpopulation of patients with advanced HCC in a multicenter large retrospective cohort in a real-world setting, analyzed by an independent biostatistician according to a predefined analyses plan. We believe that our results demonstrate that HAIC is a more suitable treatment strategy than sorafenib in advanced HCC patients with MVI, but without EHM.

The present retrospective study subdivided patients with advanced HCC into four groups according to the absence or presence of both MVI and EHM. As far as we knew, only few trials evaluated the outcomes of advanced HCC in this way. In addition to the results of cohort 1 , we also found that HAIC conferred a significantly better survival benefit in patients with both MVI and EHM (cohort 3). In advanced HCC patients with EHM, but without MVI (cohort 4), the OS of HAIC was not significantly longer than that of sorafenib. Although our cohort had 
treatment selection biases between HAIC and sorafenib in both cohort 3 and 4 (cohort 3 , HAIC: 48 patients, sorafenib: 167 patients; cohort 4, HAIC: 22 patients, sorafenib: 424 patients), HAIC might have potential as a treatment choice in advanced HCC patients with MVI, no matter whether EHM was absent or present.

Our results concur with the findings from previous small sample size prospective and retrospective studies that compared HAIC and sorafenib treatment in patients with advanced HCC with MVI and showed that the OS and objective response of HAIC were significantly better than that of sorafenib [17-20]. In a recent retrospective study in China, Lyu et al. [21] reported that HAIC of FOLFOX improved OS compared with sorafenib in a large number of patients with advanced HCC, including more than 50\% with MVI. MVI is commonly found during the natural clinical course of HCC, and its occurrence reduces patient survival. The growth pattern of MVI is predominantly characterized by invasion from intrahepatic nodules that progresses toward the central trunk (i.e., from a branch of the portal vein to the main portal vein, or from the hepatic vein to the inferior vena cava) [22,23]. This highlights that MVI of HCC is a unique disease phenotype that should not be regarded as typical metastasis of other malignancies. Taken together, HAIC, which delivers high concentrations of anticancer agents directly to the liver, represents a specific and beneficial treatment approach for advanced HCC patients with MVI.

Three randomized controlled trials conducted in Japan and China compared sorafenib plus HAIC and sorafenib $[15,16,24]$. The primary outcome of the phase III trial from China concluded that combined treatment with HAIC and sorafenib dramatically improved OS in patients with advanced HCC and portal vein invasion, irrespective of the presence of EHM [16]. The subanalysis of the SILIUS trial from Japan also confirmed that HAIC has additive effectiveness to sorafenib, which is standard systemic therapy, in advanced HCC patients with main portal invasion [15]. Taken together, it is assumed that sorafenib plus HAIC has beneficial effects, particularly in patients with advanced HCC with MVI and EHM, due to the additive effect of both treatments, as HAIC and sorafenib affect systemic and intrahepatic lesions with MVI, respectively. However, it remains controversial whether the clinical outcomes of HAIC plus sorafenib are more beneficial than those of HAIC monotherapy in advanced HCC patients with MVI, but without EHM. The outcomes of these trials did not evaluate the treatment outcome of HAIC monotherapy, since HAIC and sorafenib were not directly compared [15, 16]. The majority of patients suffer specific harmful adverse events during sorafenib therapy, such as hand-foot-skin reaction, rash, or hypertension $[3,4,25]$. A comparison of HAIC plus sorafenib with HAIC is required in future clinical trials using a subpopulation of patients with advanced HCC with MVI, but without EHM.

Over the past decades, various regimens and technical approaches of HAIC have been reported from East Asia, particularly Japan [10-13, 15, 16, 19, 21, 24, 26]. However, standardization of the methodology of HAIC has not yet been established. For example, the port system is necessary for the 5FU + CDDP regimen due to continuous injection of $5 \mathrm{FU}$ for $120 \mathrm{~h}$ [15]. On the other hand, a CDDP monotherapy regimen could be conducted by the temporary insertion of a catheter to hepatic arteries each time [24]. Subgroup analyses of the present study compared three common regimens of HAIC in a Japanes population. From our results, we were unable to identify the most beneficial HAIC regimen. Although not common in Japan, HAIC of FOLFOX is a major regimen in China $[16,21]$. Standardization of the methodology of HAIC is essential to generalize HAIC from a local treatment strategy used in East Asia to a treatment used worldwide. The results from our study as well as other studies indicate that combined use of 5FU and platinum-containing drugs may be the optimum HAIC regimen.

In 2016, the REFLECT trial demonstrated that lenvatinib was non-inferior to sorafenib in terms of OS. Furthermore, lenvatinib increased progression-free survival, time to progression, and objective response [5]. Lately, lenvatinib, a second tyrosine kinase inhibitor, with potential 
for being applied as first-line therapy, has been approved and used widely all over the world for advanced HCC $[27,28]$. In 2019, atezolizumab combined with bevacizumab for first-line treatment of patients with advanced HCC prolonged OS and progression-free survival compared with sorafenib, according to the results of IMbrave 150, which was a global phase III study [29]. The IMbrave 150 results may be practice-changing in the front-line setting of advanced HCC management. Since treatment strategies of advanced HCC will dramatically change in the near future, HAIC should be performed for more targeted subgroups of advanced HCC patients. We believe that our study could inform the selection of the population who would benefit the most, such as advanced HCC patients with MVI who fail to respond to the new front-line agent.

In conclusion, HAIC is a potential front-line treatment choice in a subpopulation of patients with advanced HCC with MVI, but without EHM. Lenvatinib, a second tyrosine kinase inhibitor as first-line therapy, has been approved worldwide for advanced HCC [5]. In addition, HAIC plus sorafenib may represent an alternate treatment option in patients with advanced HCC with MVI. Since practice changes in the treatment of advanced HCC will occur in the near future, further studies will be required to find a role for HAIC in new treatment strategies for advanced HCC. Standardization of the methodology of HAIC is also required to generalize HAIC as a standard therapy for patients with advanced HCC worldwide.

\section{Acknowledgements}

All authors are grateful to Kazufumi Yoshida and his team (SRL Medisearch Inc.) for assistance with the data management. We would also like to thank Enago (www.enago.jp) for the English language review.

\section{Statement of Ethics}

All procedures performed in studies involving human participants were in accordance with the ethical standards of the institutional and/or national research committee and with the 1964 Helsinki declaration and its later amendments or comparable ethical standards. For this type of study formal consent was not required.

The present study was approved by the Research Ethics Committees of the Kindai University Faculty of Medicine (29-196), the Graduate School of Medicine, Chiba University (2775), the Musashino Red Cross Hospital (702), the Kyoundo Hospital of the Sasaki Institute (H29-1-2), the Sapporo Kosei General Hospital (425), the Iwate Medical University (H29-95), the Mitsui Memorial Hospital (C53), the Institutional Review Boards of National Cancer Center Hospital East (2017-179), the Kanazawa University Hospital (2571-1), and the Hiroshima University Hospital (E-934).

\section{Conflict of Interest Statement}

K.U. received honoraria from Bayer and Eisai, and consulting or advisory fees from Eisai and Eli Lilly. S. Ogasawara received honoraria from Bayer, Eisai, Eli Lilly, consulting or advisory fees from Bayer, Eisai, Merck \& Co. Inc., Chugai Pharma, Eli Lilly, and research grants from Bayer and Eisai. M.I. received consulting or advisory fees from Bayer Yakuhin, Eisai, Novartis Pharma, Shire, and MSD, and research grants from Bayer Yakuhin, Kyowa Hakko Kirin, Yakult, Eli Lilly Japan, Ono Pharmaceutical, Eisai, AstraZeneca, Baxalta Japan Limited, Chugai Pharmaceutical, Bristol-Myers Squibb, Merck Serono, Nano Carrier, ASLAN Pharmaceuticals, Novartis Pharma, and Takara Bio. Y.Y. received honoraria from Bayer and an Overseas Research Fellowship from the Uehara Memorial Foundation. T.T. received a consulting or advisory role,, speakers bureau, and research funding from Bayer and Eisai. T.Y. received consulting or advisory roles, speakers' bureau, and research funding from Bayer and Eisai. S. Obi received research funding from Eisai, Otsuka pharmaceutical, and Mochida pharmaceutical. T. Ohmura received honoraria from Eisai and Bayer and speakers bureau from Bayer. T. Ohki received speakers bureau from Bayer, Eisai, STARmed, Abbvie, Shionogi, Otsuka Pharmaceu-

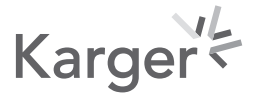


tical, and Sumitomo Dainippon Pharma. K.N. received a lecture fee from Pfizer. Y.O. received honoraria from Bayer, Eisai, and Sumitomo Dainippon Pharma. M. Kurosaki received speakers bureau from Eisai and Bayer. K.C. received a consulting or advisory fee from Abbvie, Asuka, Glaxo Smithkline, and speakers bureau from Astellas, Abbvie, Abbot, Eisai, Merck \& Co. Inc., Otuka, Olympus Medical Systems, Gilead Sciences, JIMRO, ZERIA Pharmaceutical, Daiichi Sankyo, Taisho Pharmaceutical, Sumitomo Dainippon Pharma, Takeda, Tanabe Pharmaceutical, Chugai Pharmaceutical, Bayer, Pfizer, Bristol-Myers Squibb, Miyarisan Pharmaceutical, and Yakult Pharmaceutical Industry. S.K. received a consulting or advisory role, speakers bureau, and research funding from Bayer and Eisai. N.I. received speakers bureau from Eisai and Bayer. N.K. received honoraria, a consulting or advisory role, and research funding from Bayer and Eisai. M. Kudo received honoraria from Bayer, Eisai, Merck \& Co. Inc., and Ajinomoto, consulting or advisory frees from Kowa, Merck \& Co. Inc., Bristol-Myers Squibb, Chugai Pharmaceutical, and Taiho Pharmaceutical, and research grants from Chugai Pharmaceutical, Otuka, Takeda, Sumitomo Dainippon Pharma, Daiichi Sankyo, Merck \& Co., Eisai, Bayer, and Abbvie. M.O. received honoraria from Bayer, Boehringer Ingelheim, Bristol-Myers Squibb, Otsuka, Astellas, Gilead Science, Chugai Pharmaceutical, Mitsubishi Tanabe, Kyorin, Merck Sharp and Dohme, Sumitomo Dainippon, Vertex Pharmaceutical, Takeda, Merck Serono, and Zeria. The other authors involved in this study have nothing to declare regarding funding or conflicts of interest.

\section{Funding Sources}

This study was supported by Bayer.

\section{Author Contributions}

All authors approved the final version of this manuscript. K.U., S. Ogasawara, and M.I. were involved in data acquisition, study design, and drafting of the manuscript. Y.Y., T.T., T.Y., S. Obi., T. Ohki, and H.A. were involved in data acquisition, study design, and critical revision of the manuscript. S.S., T. Ohmura, H.K., Y.O., and M.T. were involved in data acquisition. K.N. was involved in data analysis and interpretation. M.K., K.C., S.K., N.I., N.K., M.K., and M.O. supervised the study.

\section{References}

1 Torre LA, Bray F, Siegel RL, Ferlay J, Lortet-Tieulent J, Jemal A. Global cancer statistics, 2012. CA Cancer J Clin. 2015 Mar;65(2):87-108.

2 Llovet JM, Zucman-Rossi J, Pikarsky E, Sangro B, Schwartz M, Sherman M, et al. Hepatocellular carcinoma. Nat Rev Dis Primers. 2016 Apr;2(1):16018.

3 Llovet JM, Ricci S, Mazzaferro V, Hilgard P, Gane E, Blanc JF, et al.; SHARP Investigators Study Group. Sorafenib in advanced hepatocellular carcinoma. N Engl J Med. 2008 Jul;359(4):378-90.

4 Cheng AL, Kang YK, Chen Z, Tsao CJ, Qin S, Kim JS, et al. Efficacy and safety of sorafenib in patients in the AsiaPacific region with advanced hepatocellular carcinoma: a phase III randomised, double-blind, placebocontrolled trial. Lancet Oncol. 2009 Jan;10(1):25-34.

5 Kudo M, Finn RS, Qin S, Han KH, Ikeda K, Piscaglia F, et al. Lenvatinib versus sorafenib in first-line treatment of patients with unresectable hepatocellular carcinoma: a randomised phase 3 non-inferiority trial. Lancet. 2018 Mar;391(10126):1163-73.

6 Kudo M, Trevisani F, Abou-Alfa GK, Rimassa L. Hepatocellular carcinoma: therapeutic guidelines and medical treatment. Liver Cancer. 2016 Nov;6(1):16-26.

7 Ikeda M, Morizane C, Ueno M, Okusaka T, Ishii H, Furuse J. Chemotherapy for hepatocellular carcinoma: current status and future perspectives. Jpn J Clin Oncol. 2018 Feb;48(2):103-14.

8 Kudo M, Matsui O, Izumi N, Iijima H, Kadoya M, Imai Y, et al.; Liver Cancer Study Group of Japan. JSH Consensusbased clinical practice guidelines for the management of hepatocellular carcinoma: 2014 update by the Liver Cancer Study Group of Japan. Liver Cancer. 2014 Oct;3(3-4):458-68.

9 Bartkowski R, Berger MR, Aguiar JL, Henne TH, Dörsam J, Geelhaar GH, et al. Experiments on the efficacy and toxicity of locoregional chemotherapy of liver tumors with 5-fluoro-2'-deoxyuridine (FUDR) and 5-fluorouracil (5-FU) in an animal model. J Cancer Res Clin Oncol. 1986;111(1):42-6.

10 Nouso K, Miyahara K, Uchida D, Kuwaki K, Izumi N, Omata M, et al.; Liver Cancer Study Group of Japan. Effect of hepatic arterial infusion chemotherapy of 5-fluorouracil and cisplatin for advanced hepatocellular carcinoma in the Nationwide Survey of Primary Liver Cancer in Japan. Br J Cancer. 2013 Oct;109(7):1904-7.

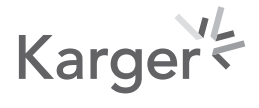




\begin{tabular}{l|l|}
\hline Liver Cancer 2020;9:583-595 \\
\hline DOI: 10.1159/000508724 & $\begin{array}{l}\text { @ 2020 The Author(s). Published by S. Karger AG, Basel } \\
\text { www.karger.com/lic }\end{array}$ \\
\hline
\end{tabular}

Ueshima et al.: HAIC versus Sorafenib in Patients with Advanced HCC

11 Chung YH, Song IH, Song BC, Lee GC, Koh MS, Yoon HK, et al. Combined therapy consisting of intraarterial cisplatin infusion and systemic interferon-alpha for hepatocellular carcinoma patients with major portal vein thrombosis or distant metastasis. Cancer. 2000 May;88(9):1986-91.

12 Yoshikawa M, Ono N, Yodono H, Ichida T, Nakamura H. Phase II study of hepatic arterial infusion of a finepowder formulation of cisplatin for advanced hepatocellular carcinoma. Hepatol Res. 2008 May;38(5):47483.

13 Ikeda M, Okusaka T, Furuse J, Mitsunaga S, Ueno H, Yamaura H, et al. A multi-institutional phase II trial of hepatic arterial infusion chemotherapy with cisplatin for advanced hepatocellular carcinoma with portal vein tumor thrombosis. Cancer Chemother Pharmacol. 2013 Aug; 72(2):463-70.

14 Ando E, Tanaka M, Yamashita F, Kuromatsu R, Yutani S, Fukumori K, et al. Hepatic arterial infusion chemotherapy for advanced hepatocellular carcinoma with portal vein tumor thrombosis: analysis of 48 cases. Cancer. 2002 Aug;95(3):588-95.

15 Kudo M, Ueshima K, Yokosuka O, Ogasawara S, Obi S, Izumi N, et al.; SILIUS study group. Sorafenib plus low-dose cisplatin and fluorouracil hepatic arterial infusion chemotherapy versus sorafenib alone in patients with advanced hepatocellular carcinoma (SILIUS): a randomised, open label, phase 3 trial. Lancet Gastroenterol Hepatol. 2018 Jun;3(6):424-32.

16 He M, Li Q, Zou R, Shen J, Fang W, Tan G, et al. Sorafenib Plus Hepatic Arterial Infusion of Oxaliplatin, Fluorouracil, and Leucovorin vs Sorafenib Alone for Hepatocellular Carcinoma With Portal Vein Invasion: A Randomized Clinical Trial. JAMA Oncol. 2019 Jul;5(7):953-60.

17 Kawaoka T, Aikata H, Hyogo H, Morio R, Morio K, Hatooka M, et al. Comparison of hepatic arterial infusion chemotherapy versus sorafenib monotherapy in patients with advanced hepatocellular carcinoma. J Dig Dis. 2015 Sep;16(9):505-12.

18 Choi JH, Chung WJ, Bae SH, Song DS, Song MJ, Kim YS, et al. Randomized, prospective, comparative study on the effects and safety of sorafenib vs. hepatic arterial infusion chemotherapy in patients with advanced hepatocellular carcinoma with portal vein tumor thrombosis. Cancer Chemother Pharmacol. 2018 Sep;82(3):46978.

19 Nakano M, Niizeki T, Nagamatsu H, Tanaka M, Kuromatsu R, Satani M, et al.; Kurume Liver Cancer Study Group of Japan. Clinical effects and safety of intra-arterial infusion therapy of cisplatin suspension in lipiodol combined with 5-fluorouracil versus sorafenib, for advanced hepatocellular carcinoma with macroscopic vascular invasion without extra-hepatic spread: A prospective cohort study. Mol Clin Oncol. 2017 Dec; 7(6): 1013-20.

20 Moriguchi M, Aramaki T, Nishiofuku H, Sato R, Asakura K, Yamaguchi K, et al. Sorafenib versus hepatic arterial infusion chemotherapy as initial treatment for hepatocellular carcinoma with advanced portal vein tumor thrombosis. Liver Cancer. 2017 Nov;6(4):275-86.

21 Lyu N, Kong Y, Mu L, Lin Y, Li J, Liu Y, et al. Hepatic arterial infusion of oxaliplatin plus fluorouracil/leucovorin vs. sorafenib for advanced hepatocellular carcinoma. J Hepatol. 2018 Jul;69(1):60-9.

22 Costentin CE, Ferrone CR, Arellano RS, Ganguli S, Hong TS, Zhu AX. Hepatocellular carcinoma with macrovascular invasion: defining the optimal treatment strategy. Liver Cancer. 2017 Nov;6(4):360-74.

23 Kudo M, Izumi N, Ichida T, Ku Y, Kokudo N, Sakamoto M, et al. Report of the 19th follow-up survey of primary liver cancer in Japan. Hepatol Res. 2016 Mar;46(5):372-90.

24 Ikeda M, Shimizu S, Sato T, Morimoto M, Kojima Y, Inaba Y, et al. Sorafenib plus hepatic arterial infusion chemotherapy with cisplatin versus sorafenib for advanced hepatocellular carcinoma: randomized phase II trial. Ann Oncol. 2016 Nov;27(11):2090-6.

25 Ogasawara S, Chiba T, Ooka Y, Kanogawa N, Saito T, Motoyama T, et al. Sorafenib treatment in Child-Pugh A and B patients with advanced hepatocellular carcinoma: safety, efficacy and prognostic factors. Invest New Drugs. 2015 Jun;33(3):729-39.

26 Yamashita T, Arai K, Sunagozaka H, Ueda T, Terashima T, Yamashita T, et al. Randomized, phase II study comparing interferon combined with hepatic arterial infusion of fluorouracil plus cisplatin and fluorouracil alone in patients with advanced hepatocellular carcinoma. Oncology. 2011;81(5-6):281-90.

27 Hiraoka A, Kumada T, Kariyama K, Takaguchi K, Atsukawa M, Itobayashi E, et al.; Real-life Practice Experts for HCC (RELPEC) Study Group, HCC 48 Group (hepatocellular carcinoma experts from 48 clinics in Japan). Clinical features of lenvatinib for unresectable hepatocellular carcinoma in real-world conditions: multicenter analysis. Cancer Med. 2019 Jan;8(1):137-46.

28 Maruta S, Ogasawara S, Ooka Y, Obu M, Inoue M, Itokawa N, et al. Potential of Lenvatinib for an expanded indication from the REFLECT trial in patients with advanced hepatocellular carcinoma. Liver Cancer. 2020;1-15.

29 Finn RS, Qin S, Ikeda M, et al. Atezolizumab plus bevacizumab in unresectable hepatocellular carcinoma. N Engl J Med. 2020;382(20):1894-1905. 